\title{
Kinetic instabilities in non-equilibrium plasma: a review of observations
}

\author{
D.A. Mansfeld \\ Institute of applied physics, Nizhny Novgorod, Russia, mda1981@appl.sci-nnov.ru
}

Resonant interaction of electromagnetic waves and energetic electrons plays an important role in the dynamics of magnetoactive plasma confined in space and laboratory magnetic traps. One of the most intriguing manifestations of such interaction is the generation of powerful electromagnetic radiation and bursts of energetic electrons from the magnetic trap due to kinetic instabilities. Kinetic instabilities are caused by the presence of positive gradients in the velocity distribution of resonant particles due to different acceleration mechanisms in space (betatron acceleration, acceleration as a result of plasma-wave turbulence and magnetic reconnection) and laboratory plasma (cyclotron heating, magnetic compression, beam heating).

Understanding of the fundamental mechanisms of generation of waves in all frequency ranges and plasma parameters is important to build a complete model of the electromagnetic background not only in the Earth's magnetosphere, but also for other planets. Field experiments, primarily satellite measurements are subject to significant restrictions because of the local nature and complexity of the measurement of spatial and temporal separation of dependencies, especially significant for non-stationary processes. In view of the universality of the physical mechanisms of generation of radiation, significant aspects of natural systems can be reproduced in the laboratory in the open magnetic configurations with magnetic mirrors. The laboratory simulation is valuable due to the fact that, firstly, in the laboratory it is possible to change the parameters of the plasma in a controlled manner, and secondly, to provide multiple repeatability. The fundamental goal of the study is to define universal mechanisms responsible for the formation of temporal regimes and dynamic spectra of stimulated emission of unstable plasma, determination of plasma emission propagation properties, and also to study the characteristics of the generated radiation impact on the plasma confinement in the trap, and the distribution of particles leaving the trap.

The opportunity to recreate different conditions for excitation and amplification of waves in plasma in a single electron cyclotron resonance (ECR) discharge pulse has been demonstrated by IAPRAS scientists, which have the priority results in this area [1-3]. The highly nonequilibrium ECR discharge plasma was created and sustained in the mirror magnetic trap by the pulsed gyrotron microwave radiation at a frequency of $37.5 \mathrm{GHz}$ at power level up to $100 \mathrm{~kW}$. At different stage of the discharge different conditions for excitation of waves are realized which are defined by the ratio fpe/fce (fpe - electron plasma frequency, fce - electron cyclotron frequency): (1) rarefied plasma at initial discharge stage (fpe $<<$ fce), when density of hot electrons exceeds density of cold fraction; (2) dense plasma at developed discharge stage (fpe $>$ fce), when density of hot particles is much less than density of cold plasma; (3) at plasma decay stage (fpe $<<$ fce), when density of hot and cold plasma fraction became the same order of magnitude (at a certain decay stage). For example, the plasma electromagnetic activity, which is realized at the advanced stage of the ECR discharge in axially symmetric magnetic trap was studied in [1], and the quasiperiodic pulsed bursts of energetic electrons at the stage of plasma decay were first observed in [3]. The results of these experiments were explained the model of the cyclotron maser [2]. The observed in experiments generation of electromagnetic radiation bursts have much in common with the natural sources of coherent cyclotron radiation - the so-called space cyclotron masers defining population energetic particles of the radiation belts, and as well as responsible for the formation of electromagnetic radiation the Earth's magnetosphere in ELF - VLF range, Jovian decametric emission and some types of solar activity. [4, 5].

In the last decade the interest in this study was renewed due the most powerful digital oscilloscopes with a broad band (up to $60 \mathrm{GHz}$ ) and high-speed ADC (up to $160 \mathrm{GS} / \mathrm{s}$ ), which in real time measure the electric field of the wave. With their help, for the first time we have the opportunity to register with the necessary temporal resolution low-power broadband electromagnetic radiation emerging from the plasma, to determine its dynamic spectrum allocated to the background of strong interference. Measuring electromagnetic field with high temporal resolution allowed to observe various dynamic spectra of electromagnetic radiation related to at least five types of kinetic instabilities [6]. The detailed analysis of electromagnetic spectra of kinetic instabilities in non-equilibrium plasma can give a valuable diagnostic information on the plasma parameters (plasma density, the characteristic energy of the emitting particles, etc.) [7].

Studying of these mechanisms in the laboratory traps has its own value because magnetic traps filled with hot non-equilibrium plasmas have a lot of practical (technology) applications in thermonuclear fusion devices, ECR multicharged ion sources, which used in accelerators of heavy particles, UV and X-ray sources, thermonuclear neutrons sources, etc. The problem is especially relevant in connection with the use of high-power microwave sources - gyrotrons, application of which for increasing of the energy input into the plasma, can achieve not only the record values of main component plasma density and temperature, but also significantly increase the anisotropy of the velocity distribution function of «warm» and «hot» electronic components. Thus there are various kinds of plasma instabilities primarily kinetic nature that with increasing density and characteristic energy of nonequilibrium particles begin to play a crucial role in both plasma confinement and energy transfer in the balance between plasma components. Significant losses of charged particles from the trap as a result of instability 
leads to the generation of bremsstrahlung X-ray radiation, which is absorbed by the coolant of the magnetic systems (cryomagnet) and increases the thermal load. For example, the ECR ion sources powerful emission of highenergy electrons from the traps can lead to disturbance of the ambipolar potential and undesirable oscillations of the currents of multiply charged ions [8]. In this sense, the cyclotron instability, which is inherent with ECR heating systems, in natural way (and sometimes very significantly!) limits the achievement of the record parameters of the source. Therefore, the problem of achieving the record parameter settings (mostly, for sources of multiply charged ions) is directly related to the controlling of the dynamics of energetic electrons fraction during ECR heating. The problem of formation of suprathermal electrons is also important in understanding plasma processes in thermonuclear fusion devices. For example, despite low density the fraction of hot electrons plays an essential role in ECR plasma startup at the axially symmetric magnetic mirror device gas dynamic trap (GDT) (Budker Institute, Novosibirsk) [9]. Also, intense bursts of coherent microwave radiation were detected in plasma of MegaAmp Spherical Tokamak (MAST) during edge localized mode (ELM) activity [10]. The most obvious reason of this phenomena is the electron acceleration by inductive electric fields during ELM activity.

Study of the formation mechanism of plasma emissions, may explain from the unified point of view a number of important phenomena occurring in the magnetospheres of Earth and other planets. For the laboratory plasma (ECR ion sources, ECR heating setups for thermonuclear fusion) the stabilization of kinetic plasma instabilities looks especially promising, since the electronic fraction with a non-equilibrium velocity distribution is naturally formed during the ECR plasma heating.

The author thanks to all colleagues from Institute of Applied Physics, Budker Institute and Jyvaskyla University involved in this study.

\section{References}

1. A.V. Vodopyanov, S.V. Golubev, A.G. Demekhov, V.G. Zorin, D.A. Mansfeld, S.V. Razin, V.Yu. Trakhtengerts. Laboratory modeling of nonstationary processes in space cyclotron masers: First results and prospects. // Plasma Physics Reports 2005, Volume 31, Issue 11, pp 927-937.

2. A.G. Shalashov, A.V. Vodopyanov, S.V. Golubev, A.G. Demekhov, V.G. Zorin, D.A. Mansfeld, S.V. Razin. Maser based on cyclotron resonance in a decaying plasma. // JETP Letters. 2006, v.84, is.6, 314-319.

3. A.V. Vodopyanov, S.V. Golubev, A.G. Demekhov, V.G. Zorin, D.A. Mansfeld, S.V. Razin, A.G. Shalashov. Observation of pulsed fast electron precipitations and the cyclotron generation mechanism of burst activity in a decaying ECR discharge plasma. // JETP. 2007, v. 104, is.2, p.296-306.

4. P.A. Bespalov and V.Yu Trakhtengerts. in Reviews of Plasma Physics, edited by Leontovich M. A., Vol. 10 (Consultants Bureau, New York) 1986.

5. V.Y. Trakhtengerts and M.J. Rycroft. Whistler and Alfven Mode Cyclotron Masers in Space // (Cambridge University Press, New York) 2008.

6. M. Viktorov, D. Mansfeld, S. Golubev. Laboratory study of kinetic instabilities in a nonequilibrium mirror-confined plasma. // Europhysics Letters. 2015, 109 p.65002.

7. M.E. Viktorov, S.V. Golubev, E.D. Gospodchikov, I.V. Izotov, D.A. Mansfeld, A.G. Shalashov. On the Mechanism of Energetic Electron Losses from the Magnetic Mirror Trap at the ECR Discharge Startup // Radiophysics and Quantum Electronics. 2013. V.56(4). P.216-227.

8. O. Tarvainen, I. Izotov, D. Mansfeld, V. Skalyga, S. Golubev, T. Kalvas, H. Koivisto, J. Komppula, R. Kronholm, $J$. Laulainen, $V$. Toivanen. Beam current oscillations driven by cyclotron instabilities in a minimum-B electron cyclotron resonance ion source plasma. // Plasma Sources Sci. Technol. 201423025020

9. D.V. Yakovlev, A. G. Shalashov, E. D. Gospodchikov A.L. Solomakhin, V.Ya. Savkin, P.A. Bagryansky. Electron cyclotron plasma startup in the GDT experiment // Nuclear Fusion. 2016. V. 57. P. 016033.

10. S. J. Freethy, K. G. McClements, S. C. Chapman, R. O. Dendy, W. N. Lai, S. J. P. Pamela, V.F. Shevchenko, R. G. L. Vann. Electron Kinetics Inferred from Observations of Microwave Bursts During Edge Localized Modes in the Mega-Amp Spherical Tokamak. // Phys. Rev. Lett. 114, 125004. 